\title{
Point spread function reconstruction validated using on-sky CANARY data in multiobject adaptive optics mode
}

\author{
O.A. Martin ${ }^{\mathrm{a}}$, C.M. Correia ${ }^{\mathrm{a}}$, E. Gendron ${ }^{\mathrm{b}}$, G. Rousset ${ }^{\mathrm{b}}$, D. Gratadour ${ }^{\mathrm{b}}$, F. Vidal ${ }^{\mathrm{b}}$, T.J. \\ Morris $^{\mathrm{c}}$, A.G. Basden ${ }^{\mathrm{c}}$, R.M. Myers ${ }^{\mathrm{c}}$, B. Neichel ${ }^{\mathrm{a}}$, and T. Fusco ${ }^{\mathrm{a}, \mathrm{d}}$ \\ ${ }^{a}$ Aix Marseille Université, CNRS, LAM (Laboratoire d'Astrophysique de Marseille) UMR \\ 7326, 13388 Marseille, France \\ ${ }^{\mathrm{b}}$ LESIA, Obs. Paris, CNRS, UPMC, Univ. Paris Diderot, 5 pl. Janssen, 92190 Meudon, France \\ ${ }^{\mathrm{c}}$ Centre for Advanced Instrumentation, Durham Univ., South Road, Durham, DH1 3LE, UK \\ ${ }^{d}$ ONERA (Office National d'Etudes et de Recherches Aérospatiales), B.P.72, F-92322 \\ Châtillon, France
}

\begin{abstract}
CANARY is an open-loop tomographic adaptive optics (AO) demonstrator that was designed for use at the $4.2 \mathrm{~m}$ William Herschel Telescope (WHT) in La Palma. Gearing up to extensive statistical studies of high redshifted galaxies surveyed with Multi-Object Spectrographs (MOS), the demonstrator CANARY as been designed to tackle technical challenges related to open-loop Adaptive-Optics (AO) control with mixed Natural Guide Star (NGS) and Laser Guide Star (LGS) tomography.

We have developed a Point Spread Function (PSF)-Reconstruction algorithm dedicated to MOAO systems using system telemetry to estimate the PSF potentially anywhere in the observed field, a prerequisite to deconvolve AO-corrected science observations in Integral Field Spectroscopy (IFS). Additionally the ability to accurately reconstruct the PSF is the materialization of the broad and fine-detailed understanding of the residual error contributors, both atmospheric and opto-mechanical.

In this paper we compare the classical PSF-r approach from Véran $\left({ }^{1}\right)$ that we take as reference on-axis using the truth-sensor telemetry to one tailored to atmospheric tomography by handling the off-axis data only.

We've post-processed over 450 on-sky CANARY data sets with which we observe $92 \%$ and $88 \%$ of correlation on respectively the reconstructed Strehl Ratio (SR)/Full Width at Half Maximum (FWHM) compared to the sky values. The reference method achieves $95 \%$ and $92.5 \%$ exploiting directly the measurements of the residual phase from the CANARY Truth Sensor (TS).
\end{abstract}

Keywords: Multi-object adaptive optics, CANARY, PSF reconstruction

\section{INTRODUCTION}

For studies of formation and evolution of early galaxies, multiplexed observations of several objects need be carried out at once using integral field spectrographs over a large Field-of-View (FoV). The need to correct only a limited number of discrete directions over the field led to the concept of Multi-object adaptive optics (MOAO) which uses separate narrow field wavefront correctors for each of several objects. To solve optical issues encountered on both wide FoV and large dimension systems, the MOAO concept consists in putting Wave-Front Sensors (WFS) ahead the Deformable Mirror (DM) in the optical path. We adopt thus an open-loop configuration since there is no longer feedback between DM and WFS.

Such a design has been proposed for EAGLE $\left({ }^{2}\right)$, the first multi-object Integral Field Units (IFU) proposed for the European-Extremely Large Telescope (E-ELT) $\left({ }^{3}\right)$, with a large FoV of 5 to 10 arc-minutes required for science observations $\left({ }^{4}\right)$.

Further author information: olivier.martin@lam.fr 
Today, MosAIC is considered the new MOAO-assisted multi-object IFU for the E-ELT $\left(^{5}\right)$. The development of MosaIC will include a High-Multiplex Mode (HMM), seeing-limited or coarsely-sampled using the Ground Layer Adaptive Optics (GLAO) available on the E-ELT using the M4 $\left({ }^{6}\right)$ pre-focal adaptive mirror, for observing from 100 to 250 objects. In addition, it will also include a High-Definition Mode (HDM) for observing from 10 to 20 faint galaxies with MOAO-corrected IFUs.

The feasibility of designing such a multi-channel instrument has to be demonstrated on-sky mainly for two reasons. The first relates to the reliability and high-fidelity control of DMs in open-loop configuration. Whereas the recursive nature of a closed-loop hides potential inaccuracies in the linear relationship between WFS and DM - the interaction matrix - in open-loop the absence of feedback puts to light ever-present deviations, DM creeping, variable gains, hysteresis to cite only a few. We build on the past experiments which have demonstrated operational open-loop DM control, e.g. Volts,${ }^{7}$ Villages, ${ }^{8}$ CanarY ${ }^{9}$ and Raven. ${ }^{10}$

The second point relates to atmospheric tomography using both NGSs and LGSs, blending signals of different nature into a single estimation step.

In order to progress on these topics CANARY has been designed by an international consortium, involving the LESIA and the Durham University starting as soon as 2007. The demonstrator was installed in 2010 at the William Herschel Telescope (WHT) at La Palma, in the Canary islands. It has provided proof of our capability to handle the open-loop configuration and specify the on-sky performance, either using NGSs only $\left({ }^{11}\right)$, or mixed NGS/LGS $\left({ }^{12}\right)$.

In Sect. 2 we provide a simplified analytic expression of the residual phase in the science directions. With it we can quantify the main contributors to the residuals, as tomography or system bandwidth. A successful attempt at PSF reconstruction in MOAO will represent a crucial milestone for a comprehensive understanding of the error budget.

In Sect. 3 laying out two methods to reconstruct the PSF:

i) defining the analytic expression of the Optical Transfer Function (OTF), computed from off-science guide-star directions telemetry. It relies on the tomographic reconstructor and on calibration matrices - the same that are used during real-time operation - leading us to dub the method RTC-based PSFR.

ii) a reference method that uses Truth Sensor (TS) telemetry looking at bright science objects and thus directly measuring the residual phase. The implementation is very similar to the one proposed by Véran $\left({ }^{1}\right)$, with slight modifications required for MOAO systems. In normal operational conditions such sensor won't be usable since MOAO systems will generally observe very faint targets.

The comparison is made on over 450 on-sky CANARY data sets, acquired in September 2013. We report in Sect. 4, Strehl ratio (SR) and Full Width at Half Maximum (FWHM) evaluated on reconstructed PSF as function of sky quantities. We provide a statistical analysis to evaluate biases and variances using the RTC-based and TS-based method. We conclude in Sect. 5

\section{MOAO SYSTEM MODELING}

\subsection{Analytic expression of the residual phase}

We illustrate in Fig. 1 a schematic diagram of a single science channel MOAO system with two GS. Each of these sensing channels has a WFS, assumed to be a Shack-Hartman; it measures the uncompensated phase $\boldsymbol{a}_{\boldsymbol{\alpha} j}$ in a direction $\boldsymbol{\alpha}_{j}$. This WFS delivers noisy slopes assumed to be a linear function of the input phase through $\mathbf{G}$ - the Zernike interaction matrix, calibrated on the bench, which gives the WFS slopes from the Zernike modal coefficients according to

$$
s_{\alpha}=\mathrm{Ga} \boldsymbol{a}_{\| \alpha}+\eta_{\alpha}+r_{\alpha},
$$

with $s_{\boldsymbol{\alpha}}$ the concatenation of WFS measurements, $\boldsymbol{a}_{\| \boldsymbol{\alpha}}$ the Zernike modes corresponding to the parallel phase, $\boldsymbol{\eta}_{\boldsymbol{\alpha}}$ and $\boldsymbol{r}_{\boldsymbol{\alpha}}$ the additive measurement noise and aliasing respectively.

The WFS slopes $\boldsymbol{s}_{\boldsymbol{\alpha}}$ are used to estimate the slopes $\widehat{\boldsymbol{s}}_{\boldsymbol{\beta}}$ in direction $\beta$ through the tomographic reconstructor $\mathbf{R}$ defined in slopes space $\left({ }^{13}\right)$. The latter is calibrated on-sky. The estimation of the on-axis phase $\widehat{\boldsymbol{a}}_{\| \boldsymbol{\beta}}$ is then 


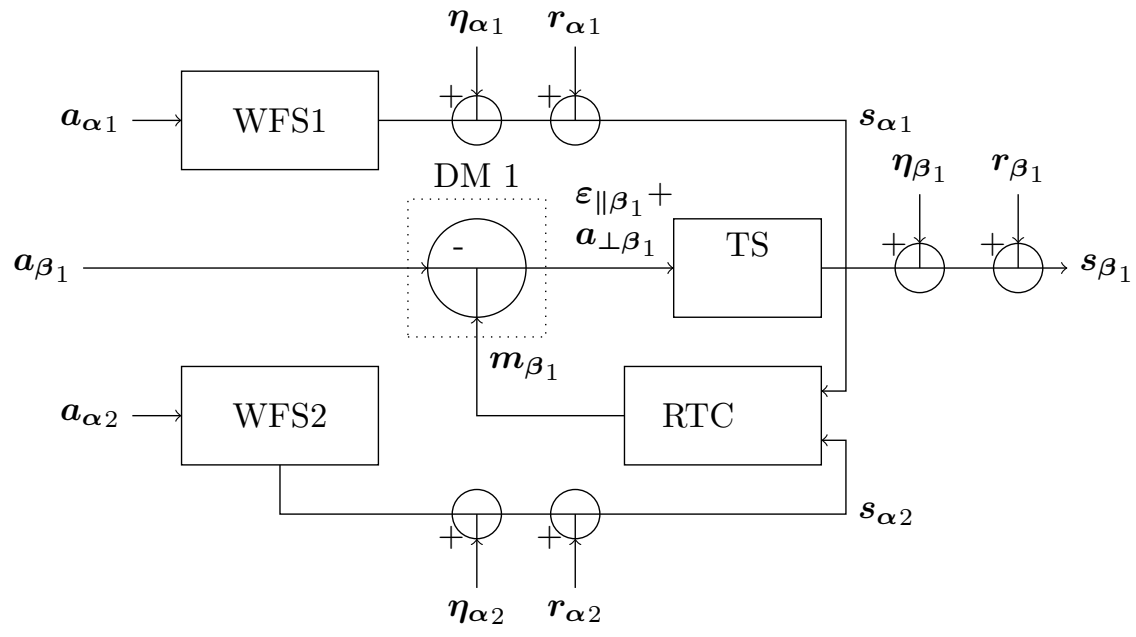

Figure 1: Description by block functions of a elementary MOAO system using two sensing WFS to compensate the turbulence into one science direction. See text for a more complete description.

given by:

$$
\begin{aligned}
\widehat{a}_{\| \beta} & =\mathrm{CR} s_{\alpha} \\
& \simeq \mathrm{CRGa} a_{\| \alpha}+\mathrm{CR} \eta_{\alpha}+\mathrm{CR} r_{\alpha}
\end{aligned}
$$

where we use $\mathbf{C}$ - the pseudo-inverse of $\mathbf{G}$ - to reconstruct the pupil-plane phase out of slopes. We further assume $\mathbf{C G} \simeq \mathbf{I}$, the identity matrix.

The DM produces a corrective phase $\boldsymbol{m}_{\boldsymbol{\beta}}$, updated iteratively from $\widehat{\boldsymbol{a}}_{\| \boldsymbol{\beta}}$. To reproduce the total system fractional delay $1+\Delta_{t}$, with $\Delta_{t}$ calibrated on on the bench to be a fraction of 0.45 of a frame, we combine values of $\widehat{\boldsymbol{a}}_{\| \boldsymbol{\beta}}$ acquired at time $t-1$ and $t-2$. Mathematically, we have

$$
\boldsymbol{m}_{\boldsymbol{\beta}}(t)=(1-g) \times \boldsymbol{m}_{\boldsymbol{\beta}}(t-1)+g\left(\Delta_{t} \widehat{\boldsymbol{a}}_{\| \boldsymbol{\beta}}(t-2)+\left(1-\Delta_{t}\right) \widehat{\boldsymbol{a}}_{\| \boldsymbol{\beta}}(t-1)\right) .
$$

Applying the Z-transform to Eq. 3 gives

$$
\tilde{\boldsymbol{m}}_{\boldsymbol{\beta}}(z)=\tilde{h}_{\mathrm{ol}}(z) \tilde{\widehat{\boldsymbol{a}}}_{\| \boldsymbol{\beta}}(z),
$$

where $z=e^{-2 i \pi \nu / \nu_{e}}, \nu_{e}$ the temporal sampling frequency and $\tilde{h}_{\mathrm{ol}}(z)$ the controller transfer function defined as

$$
\tilde{h}_{\mathrm{ol}}(z)=g \times \frac{\Delta_{t}+\left(1-\Delta_{t}\right) z}{z(z-1+g)} .
$$

We can now ascribe the phase residual as

$$
\varepsilon_{\beta}=\varepsilon_{\| \beta}+a_{\perp \beta},
$$

where $\varepsilon_{\| \boldsymbol{\beta}}$ is the in-band residual leftover after DM compensation

$$
\varepsilon_{\| \beta}=a_{\| \beta}-m_{\beta} .
$$

whereas $\boldsymbol{a}_{\perp \boldsymbol{\beta}}$ is the DM fitting error.

Combining Eqs. 2 4, 6 and 7, the Z-transform of $\boldsymbol{\varepsilon}_{\boldsymbol{\beta}}$, noted $\tilde{\varepsilon}_{\boldsymbol{\beta}}(z)$, becomes

$$
\tilde{\varepsilon}_{\boldsymbol{\beta}}=\tilde{\boldsymbol{a}}_{\| \boldsymbol{\beta}}-\tilde{h}_{\mathrm{ol}} \mathbf{C R G} \tilde{\boldsymbol{a}}_{\| \boldsymbol{\alpha}}-\tilde{h}_{\mathrm{ol}} \mathbf{C R} \tilde{\eta}_{\boldsymbol{\alpha}}-\tilde{h}_{\mathrm{ol}} \mathbf{C R} \tilde{r}_{\boldsymbol{\alpha}}+\tilde{\boldsymbol{a}}_{\perp \boldsymbol{\beta}} .
$$

where the term $\tilde{\boldsymbol{a}}_{\| \boldsymbol{\beta}}-\tilde{h}_{\mathrm{ol}} \mathbf{C R G} \tilde{\boldsymbol{a}}_{\| \boldsymbol{\alpha}}$ is the aniso-servo error that combines both tomographic reconstruction and system temporal delay errors. 


\section{Table 1: Notation}

\begin{tabular}{|c|c|}
\hline \multicolumn{2}{|r|}{ Science directions $\beta$} \\
\hline$a_{\| \beta}$ & Atmospheric parallel phase on which the system is acting \\
\hline$a_{\perp \beta}$ & Atmospheric high spatial frequencies not corrected \\
\hline$\varepsilon_{\beta}$ & Residual modes \\
\hline$\varepsilon_{\| \beta}$ & residual of the DM correction \\
\hline$m_{\beta}$ & DM modes \\
\hline$s_{\beta}$ & Concatenation of all WFS measurements in science directions \\
\hline$\eta_{\beta}$ & Noise contribution into WFS measurements \\
\hline$r_{\beta}^{\varepsilon}$ & Aliasing contribution into WFS measurements in loop engaged \\
\hline$r_{\beta}$ & Aliasing contribution into WFS measurements in loop disengaged \\
\hline \multicolumn{2}{|r|}{ Sensing directions $\alpha$} \\
\hline$s_{\alpha}$ & Concatenation of all WFS measurements in sensing directions \\
\hline$a_{\| \alpha}$ & Measured modes by WFS in sensing directions \\
\hline$\eta_{\alpha}$ & Noise contribution into WFS measurements \\
\hline$r_{\alpha}$ & Aliasing contribution into WFS measurements \\
\hline \multicolumn{2}{|r|}{ Matrices } \\
\hline $\mathbf{C}$ & Zernike reconstruction matrix (slopes to Zernike): calibrated on bench \\
\hline G & Zernike interaction matrix (Zernike to slopes): calibrated on bench \\
\hline $\mathbf{R}$ & Tomographic reconstructor (slopes $\boldsymbol{\alpha}$ to slopes $\boldsymbol{\beta}$ ): calibrated on-sky using the Learn\& Apply approach \\
\hline $\boldsymbol{Z}_{i}(\boldsymbol{r})$ & $i^{\text {th }}$ Zernike polynomials. \\
\hline \multicolumn{2}{|r|}{ System } \\
\hline$g$ & Loop gain \\
\hline$\Delta_{t}$ & Latency of the system in frames calibrated on bench at 0.45 frame. \\
\hline$\nu_{e}$ & Sampling frequency of the RTC, $150 \mathrm{~Hz}$ \\
\hline \multicolumn{2}{|r|}{ Miscellaneous } \\
\hline$\langle\boldsymbol{x}\rangle$ & Average of $\boldsymbol{x}$ \\
\hline$\Sigma_{\boldsymbol{x} y}$ & Cross-covariance matrix of $\boldsymbol{x}$ and $\boldsymbol{y}$ \\
\hline$\widehat{\boldsymbol{x}}$ & Estimation of $\boldsymbol{x}$ \\
\hline$\tilde{\boldsymbol{x}}(z)$ & Z-transform of $\boldsymbol{x}(t)$ \\
\hline
\end{tabular}

For on-sky CANARY performance diagnostic purposes, we have split this aniso-servo error into a sum of two components: a pure tomographic error plus a pure temporal error. We define the tomographic error as follows:

$$
\tilde{\boldsymbol{e}}_{\| \beta}=\tilde{\boldsymbol{a}}_{\| \boldsymbol{\beta}}-\mathrm{CRG} \tilde{\boldsymbol{a}}_{\| \boldsymbol{\alpha}},
$$

which allows us to rewrite Eq. 8 as

$$
\tilde{\varepsilon}_{\boldsymbol{\beta}}=\underbrace{\tilde{e}_{\| \boldsymbol{\beta}}}_{\text {Tomography }}+\underbrace{\tilde{h}_{\mathrm{rej}} \mathbf{C R G} \tilde{\boldsymbol{a}}_{\| \boldsymbol{\alpha}}}_{\text {Servo-Lag }}-\underbrace{\tilde{h}_{\mathrm{ol}} \mathbf{C R} \tilde{\eta}_{\alpha}}_{\text {Noise }}-\underbrace{\tilde{h}_{\mathrm{ol}} \mathbf{C R} \tilde{\boldsymbol{r}}_{\boldsymbol{\alpha}}}_{\text {Aliasing }}+\underbrace{\tilde{\boldsymbol{a}}_{\perp \boldsymbol{\beta}}}_{\text {Fitting }},
$$

where $\tilde{h}_{\text {rej }}$, represented in Fig. 2 , is the controller's rejection transfer function

$$
\tilde{h}_{\text {rej }}(z)=1-\tilde{h}_{\mathrm{ol}}(z) \text {. }
$$

If we assume all the terms involved in Eq. 10 are independent, we get the residual phase WF error $\sigma_{\varepsilon}^{2}$

$$
\sigma_{\varepsilon}^{2} \simeq \sigma_{\text {Tomography }}^{2}+\sigma_{\text {Servo }}^{2}+\sigma_{\text {Noise }}^{2}+\sigma_{\text {Alias }}^{2}+\sigma_{\text {Fitting }}^{2},
$$


where the WF error is given by summing over the variance of individual modes. To obtain Eq. 12 from Eq. 10 the following approximations have been done:

- We have split the aniso-servo error as a sum of a tomographic error and a pure temporal one. We have thus neglected the correlation between $\tilde{\boldsymbol{e}}_{\| \boldsymbol{\beta}}$ and $\tilde{h}_{\text {rej }} \mathbf{C R G} \tilde{\boldsymbol{a}}_{\| \boldsymbol{\alpha}}$. The rejection function $\tilde{h}_{\text {rej }}$ acts as a high pas filter according to Fig. 2, the signal $\tilde{h}_{\text {rej }} \mathbf{C R G} \tilde{\boldsymbol{a}}_{\| \boldsymbol{\alpha}}$ is thus a high temporal frequency signal. According to ${ }^{14}$ the tomographic error $\tilde{\boldsymbol{e}}_{\| \boldsymbol{\beta}}$ contains a large portion of low temporal frequencies. To a first degree, considering the statistical independence of Fourier modes, it is reasonable to neglect this correlation

- For the same reasons, we neglect the correlation between the aliasing $\tilde{h}_{\mathrm{ol}} \mathbf{C R} \tilde{r}_{\boldsymbol{\alpha}}$, mostly composed by low temporal frequencies, and the servo-lag error $\tilde{h}_{\mathrm{rej}} \mathbf{C R G} \tilde{\boldsymbol{a}}_{\| \boldsymbol{\alpha}}$.

- The correlation between the aliasing $\tilde{h}_{\mathrm{ol}} \mathbf{C R} \tilde{\boldsymbol{r}}_{\boldsymbol{\alpha}}$ and the tomographic error $\tilde{\boldsymbol{e}}_{\| \boldsymbol{\beta}}$ is considered as negligible as well. The aliasing is propagated through the tomographic reconstructor and the MOAO loop $\tilde{h}_{\mathrm{ol}}$, we can consider it as a residual, as the tomographic error is. We consider thus this cross-correlation between residual as negligible relative to the main errors in Eq. 12.

- We further neglect the correlation between the high order modes $\tilde{\boldsymbol{a}}_{\perp \boldsymbol{\beta}}$ above the DM cut-off frequency and the parallel ones included into the tomographic and servo-lag errors.

- We assume the DM is well represented by Zernike modes, using the Zernike interaction matrix calibrated on the bench.

In Sect. 4.1, we show how our modelling of $\varepsilon_{\boldsymbol{\beta}}$ fits quite well to the measurements over a large set of data. $A$ posteriori, we conclude these approximations are not critical for reconstructing the PSF at the CANARY scale.
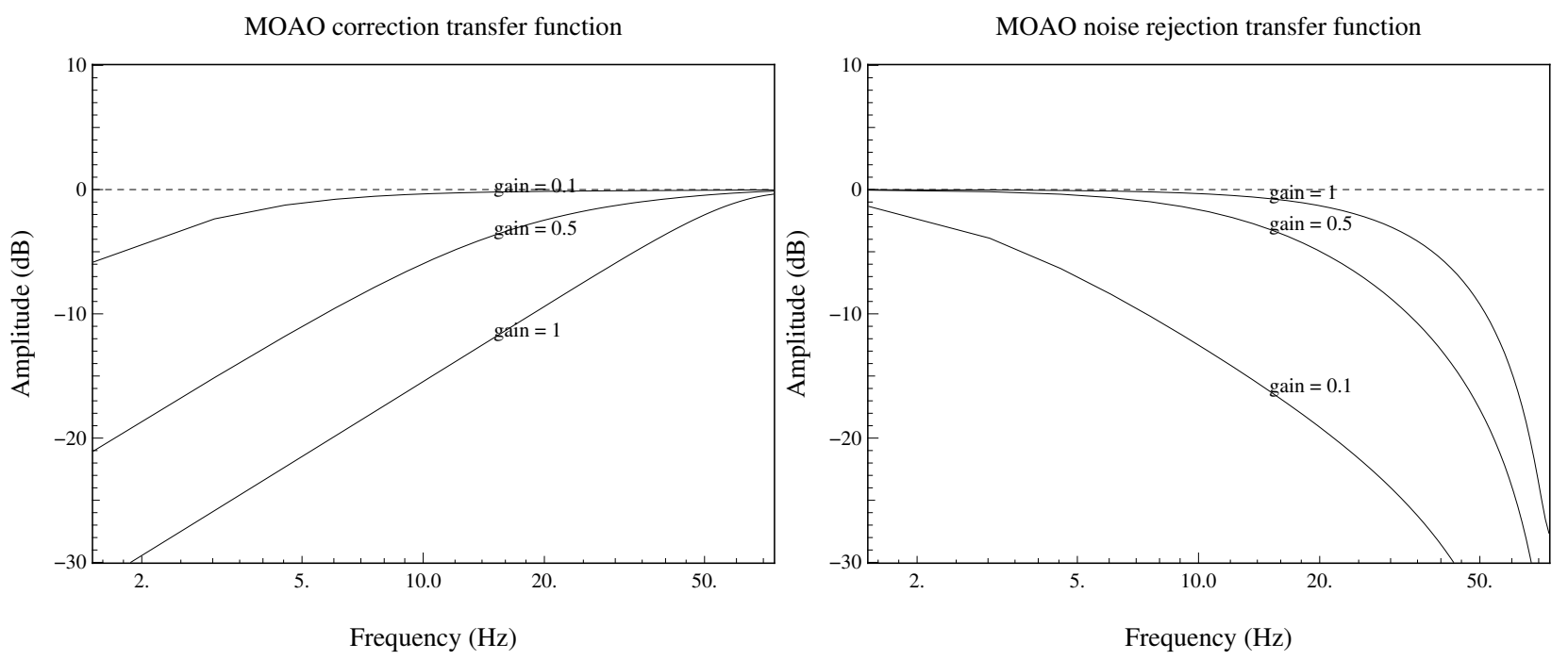

Figure 2: Bode diagrams of $\tilde{h}_{\text {rej }}$ (left) and $\tilde{h}_{\mathrm{ol}}$ (right) for three different values of gain, $\Delta_{t}=0.45$ frames and $\nu_{e}=150$ $\mathrm{Hz}$.

\subsection{Analytic expression of the TS measurements}

On CANARY we have a Truth Sensor, i.e. an extra WFS that measures the residual phase in a single science direction on-axis. In practice, observing faint targets precludes the use of such sensor to probe the residual turbulence in the science directions of interest. As a technical demonstrator, CANARY observes star asterisms composed of a bright central star, surrounded by up to two or three off-axis stars, usually separated by less than one arc-minute from the center $\left({ }^{11,12}\right)$. The CANARY TS provides an estimation of the residual phase, and is 
involved for calibration and diagnostic purposes. The vector of slopes $\boldsymbol{s}_{\boldsymbol{\beta}}$ - see Fig. 1 - provides a measurement of the residual phase with additional noise and aliasing terms

$$
s_{\beta}=\mathbf{G} \varepsilon_{\| \beta}+\eta_{\beta}+r_{\beta} .
$$

Using the Zernike reconstruction matrix $\mathbf{C}$, we get

$$
\begin{aligned}
\widehat{\varepsilon}_{\| \beta} & =\mathbf{C} s_{\beta} \\
& \simeq \varepsilon_{\| \beta}+\mathbf{C} \eta_{\beta}+\mathbf{C} r_{\beta} .
\end{aligned}
$$

Pursuing as before we get

$$
\tilde{\hat{\varepsilon}}_{\| \boldsymbol{\beta}} \simeq \underbrace{\tilde{\boldsymbol{e}}_{\| \boldsymbol{\beta}}+\tilde{h}_{\mathrm{rej}} \mathbf{C R G} \tilde{\boldsymbol{a}}_{\| \boldsymbol{\alpha}}-\tilde{h}_{\mathrm{ol}} \mathbf{C R} \tilde{\eta}_{\alpha}}_{\tilde{\varepsilon}_{\| \beta}}+\mathbf{C} \tilde{\boldsymbol{\eta}}_{\boldsymbol{\beta}}+\mathbf{C} \underbrace{\left(\tilde{\boldsymbol{r}}_{\boldsymbol{\beta}}-\tilde{h}_{\mathrm{ol}} \mathbf{R} \tilde{r}_{\boldsymbol{\alpha}}\right)}_{\tilde{r}_{\boldsymbol{\beta}}^{\epsilon}} .
$$

Equation 15 shows that the TS measures a residual aliasing term, i.e. the difference between the pure TS aliasing $\tilde{\boldsymbol{r}}_{\boldsymbol{\beta}}$ and the post-tomographic term $\tilde{h}_{\mathrm{ol}} \mathbf{R} \tilde{\boldsymbol{r}}_{\boldsymbol{\alpha}}$. We define $\boldsymbol{r}_{\boldsymbol{\beta}}^{\varepsilon}$ as this aliasing residual measured by the TS during observation as:

$$
r_{\beta}^{\varepsilon} \simeq r_{\beta}-\mathbf{R} r_{\alpha}
$$

In Eq. 16, we do not consider the temporal filtering of the off-axis aliasing as it appears in Eq. 15. Since $\tilde{h}_{\mathrm{ol}}$ operates as a low pass filter, it means we neglect the temporal high frequencies of the aliasing.

\section{MOAO PSF-R METHODS}

\subsection{OTF decomposition}

The Optical Transfer Function (OTF) of an AO system is commonly modelled as a multiplication of the telescope OTF including static aberrations, and an AO OTF of the residuals $\left({ }^{1}\right)$. This latter is then split into a in-band parallel part, $\mathrm{OTF}_{\boldsymbol{\varepsilon}_{\| \boldsymbol{\beta}}}$ over spatial frequencies the $\mathrm{AO}$ systems has acted upon and a fitting orthogonal part, $\mathrm{OTF}_{\perp \beta}$ that gathers all the spatial frequencies above the DM cut-off frequency. The final $\mathrm{OTF}_{\boldsymbol{\varepsilon}_{\boldsymbol{\beta}}}$, can be written as follows:

$$
\mathrm{OTF}_{\boldsymbol{\varepsilon}_{\beta}}=\mathrm{OTF}_{\mathrm{NCPA}_{\beta}} \times \mathrm{OTF}_{\mathrm{Static}_{\beta}} \times \mathrm{OTF}_{\perp \beta} \times \mathrm{OTF}_{\boldsymbol{\varepsilon}_{\| \beta}},
$$

where $\mathrm{OTF}_{\mathrm{NCPA}_{\beta}}$ is the telescope OTF together with the Non Common Path Aberrations (NCPA) in directions $\boldsymbol{\beta}$. It is mandatory to consider the real telescope OTF only once, which is already included into $\mathrm{OTF}_{\mathrm{NCPA}_{\beta}}$. We have to derive others terms in Eq. 17 in freeing them from this telescope OTF.

In tomographic systems the challenge is to be able to estimate $\mathrm{OTF}_{\boldsymbol{\varepsilon}_{\| \boldsymbol{\beta}}}$ without actual measurements of the residual phase on those directions. In the following, we propose to compare two different techniques:

- RTC-based method: it consists in estimating the residual phase measured by the TS when the loop is engaged. To do that, we generalize calculations presented in Sect. 2 to estimate the covariance matrix of the residual modes and then $\mathrm{OTF}_{\boldsymbol{\varepsilon}_{\| \beta}}$. See Sect. 3.2.1 for details.

The RTC-based method requires the off-axis measurements, the tomographic reconstructor $\mathbf{R}$ and the turbulence parameters as well. The latter are obtained from the Learn 3 steps approach, see paper 9909163 William Herschel site characterization using CANARY on-sky data in that conference. This is a profiling method based on the Learn \& Apply technique $\left({ }^{13}\right)$ that consists in splitting the ground and altitude layers identification. We want to emphasise that the RTC-based PSFr handles information coming from the RTC that has been either acquired during the sky observation or calibrated on the bench.

- TS-based method: this is the adaptation of the Véran's method $\left(^{1}\right)$ to MOAO systems. It will actually not be available for MOAO systems observing faint science objects. It is used in CANARY for demonstrating operational MOAO plus diagnostics as the one presented here. Using however the TS, we will be able to get the best reconstructed PSF as possible on CANARY .

This method requires the time-series of TS measurements. To manage noise and aliasing from TS, it also needs the tomographic reconstructor and a model of the aliasing covariance matrix in loop disengaged. See Sect. 3.2.2 for details. 


\subsection{Determination of $\mathrm{OTF}_{\varepsilon_{\| \beta}}$}

We use the approach from Véran $\left(^{1}\right)$. The contribution of the mirror compensation into the residual phase from the long-exposure OTF is given by :

$$
\mathrm{OTF}_{\boldsymbol{\varepsilon}_{\| \boldsymbol{\beta}}}(\boldsymbol{\rho} / \lambda)=e^{-0.5 \times \bar{D}_{\phi_{\boldsymbol{\varepsilon}} \boldsymbol{\beta}}}(\boldsymbol{\rho}),
$$

where $D_{\phi_{\boldsymbol{\varepsilon}_{\| \beta}}}$ is the residual phase structure function which when averaged over the telescope pupil becomes :

$$
\bar{D}_{\phi_{\boldsymbol{\varepsilon}_{\| \boldsymbol{\beta}}}}(\boldsymbol{\rho})=\frac{\iint_{\mathcal{P}}\left\langle\left(\phi_{\boldsymbol{\varepsilon}_{\| \boldsymbol{\beta}}}(\boldsymbol{r})-\phi_{\boldsymbol{\varepsilon}_{\| \boldsymbol{\beta}}}(\boldsymbol{\rho}+\boldsymbol{r})\right)^{2}\right\rangle P(\boldsymbol{r}) P(\boldsymbol{\rho}+\boldsymbol{r}) d \boldsymbol{r}}{\iint_{\mathcal{P}} P(\boldsymbol{r}) P(\boldsymbol{\rho}+\boldsymbol{r}) d \boldsymbol{r}},
$$

where $\mathcal{P}$ is the telescope pupil domain and $P(\boldsymbol{r})$ the pupil function. We evaluate these integrals for 36 Zernike polynomials (piston excluded) which match the spatial frequencies the CANARY DM is able to correct for $\left({ }^{11}\right)$.

$$
\phi_{\boldsymbol{\varepsilon}_{\| \boldsymbol{\beta}}}(\boldsymbol{r})=\sum_{i=2}^{36} \varepsilon_{\| \boldsymbol{\beta}} \boldsymbol{Z}_{i}(\boldsymbol{r})
$$

The structure function then becomes :

$$
\bar{D}_{\phi_{\boldsymbol{\varepsilon}_{\| \boldsymbol{\beta}}}}(\boldsymbol{\rho})=\sum_{i, j}^{n}\left\langle\varepsilon_{\| \boldsymbol{\beta}}(i) \varepsilon_{\| \boldsymbol{\beta}}(j)\right\rangle U_{i j}(\boldsymbol{\rho})
$$

where :

$$
U_{i j}(\boldsymbol{\rho})=\frac{\iint_{\mathcal{P}} P(\boldsymbol{r}) P(\boldsymbol{\rho}+\boldsymbol{r}) d \boldsymbol{r}\left(\boldsymbol{Z}_{i}(\boldsymbol{r})-\boldsymbol{Z}_{i}(\boldsymbol{r}+\boldsymbol{\rho})\right) \times\left(\boldsymbol{Z}_{j}(\boldsymbol{r})-\boldsymbol{Z}_{j}(\boldsymbol{\rho}+\boldsymbol{r})\right) d^{2} \boldsymbol{r}}{\iint_{\mathcal{P}} P(\boldsymbol{r}) P(\boldsymbol{\rho}+\boldsymbol{r}) d^{2} \boldsymbol{r}}
$$

The estimation of the PSF boils down to estimating the covariance matrix of the residual phase expressed on the Zernike modal basis $\Sigma_{\boldsymbol{\varepsilon}_{\| \boldsymbol{\beta}} \boldsymbol{\varepsilon}_{\| \boldsymbol{\beta}}}(i, j)=\left\langle\varepsilon_{\| \boldsymbol{\beta}}(i) \varepsilon_{\| \boldsymbol{\beta}}{ }^{t}(j)\right\rangle$.

\subsubsection{RTC-based PSF/R}

The RTC-based method consists in determining $\varepsilon_{\| \boldsymbol{\beta}}$ from the off-axis WFS telemetry. Eq. 10 gives an analytic expression of the Z-transform of the residual modes $\boldsymbol{\varepsilon}_{\boldsymbol{\beta}}$. In subtracting off the orthogonal part $\boldsymbol{a}_{\perp \boldsymbol{\beta}}$ and in transforming back to the real space, we get :

$$
\varepsilon_{\| \beta} \simeq e_{\| \beta}+a_{\| \beta}^{\varepsilon}+\mathbf{C R}\left(h_{\mathrm{ol}} * \eta_{\boldsymbol{\alpha}}\right)+\mathbf{C R} r_{\boldsymbol{\alpha}},
$$

where $h_{\mathrm{ol}}$ the impulsive response of the MOAO system, (.*.) the convolution product and $\boldsymbol{a}_{\| \boldsymbol{\alpha}}^{\boldsymbol{\varepsilon}}$ is the output of a first order low-pass filter defined by :

$$
\tilde{\boldsymbol{a}}_{\| \boldsymbol{\beta}}^{\varepsilon}(z)=\tilde{h}_{\mathrm{rej}}(z) \times \mathbf{C R G} \tilde{\boldsymbol{a}}_{\| \boldsymbol{\alpha}}(z),
$$

where $\tilde{h}_{\text {rej }}$ is given by Eq. 5 and Eq. 11. From Eq. 23 and Eq. 9, assuming all the terms involved in Eq. 23 are independent processes, we get the following expression of $\Sigma_{\varepsilon_{\| \beta} \varepsilon_{\| \beta}}$ :

$$
\Sigma_{\boldsymbol{\varepsilon}_{\| \beta} \boldsymbol{\varepsilon}_{\| \beta}}=\Sigma_{\boldsymbol{e}_{\| \beta} \boldsymbol{e}_{\| \beta}}+\Sigma_{\boldsymbol{a}_{\| \beta}^{\varepsilon} \boldsymbol{a}_{\| \beta}^{\varepsilon}}+\left\|\tilde{h}_{\mathrm{ol}}\right\|^{2} \times \mathbf{C R} \Sigma_{\boldsymbol{\eta}_{\alpha} \boldsymbol{\eta}_{\boldsymbol{\alpha}}} \mathbf{R}^{t} \mathbf{C}^{t}+\mathbf{C R} \Sigma_{\boldsymbol{r}_{\alpha} \boldsymbol{r}_{\alpha}} \mathbf{R}^{t} \mathbf{C}^{t},
$$

where

- $\Sigma_{\boldsymbol{e}_{\| \boldsymbol{\beta}} \boldsymbol{e}_{\| \boldsymbol{\beta}}}=\left\langle\boldsymbol{e}_{\| \boldsymbol{\beta}} \boldsymbol{e}_{\| \boldsymbol{\beta}}{ }^{t}\right\rangle$ is the tomographic error covariance matrix. Without a TS, we cannot determine the value of $\boldsymbol{e}_{\| \boldsymbol{\beta}}$. Nevertheless, from the turbulence parameters retrieved using the Learn 3 steps approach, we have a model of this covariance matrix $\left({ }^{15}\right)$. 
- $\Sigma_{\boldsymbol{a}_{\| \alpha}^{\varepsilon} \boldsymbol{a}_{\| \alpha}^{\varepsilon}}=\left\langle\boldsymbol{a}_{\| \boldsymbol{\alpha}}^{\varepsilon} \boldsymbol{a}_{\| \boldsymbol{\alpha}}^{\boldsymbol{\varepsilon}}{ }^{t}\right\rangle$ is the covariance matrix of the filtered atmospheric parallel modes by the MOAO system. It is readily derived from the time-series of the off-axis WFS measurements and parameters from the loop during the observation.

- $\Sigma_{\boldsymbol{\eta}_{\alpha} \boldsymbol{\eta}_{\alpha}}=\left\langle\boldsymbol{\eta}_{\boldsymbol{\alpha}} \boldsymbol{\eta}_{\boldsymbol{\alpha}}{ }^{t}\right\rangle$ is the noise covariance matrix of off-axis WFS telemetry, identified by a parabolic interpolation, near to a zero-delay, of temporal auto-covariance of $s_{\alpha}$

- $\Sigma_{r_{\alpha} r_{\alpha}}$ is the covariance matrix of the off-axis aliasing. It is computed from the model of the spatial covariance of slopes based on outputs from the Learn 3 steps approach.

\subsubsection{TS-based method}

The SF in Eq. 21 requires the covariance matrix of parallel modes $\Sigma_{\boldsymbol{\varepsilon}_{\| \boldsymbol{\beta}} \boldsymbol{\varepsilon}_{\| \boldsymbol{\beta}}}$ estimated from $\widehat{\boldsymbol{\varepsilon}}_{\| \boldsymbol{\beta}}(\boldsymbol{\rho}, t)$. Using Eq. 14, we get :

$$
\Sigma_{\widehat{\varepsilon}_{\| \beta} \widehat{\varepsilon}_{\| \beta}} \simeq \Sigma_{\varepsilon_{\| \beta} \varepsilon_{\| \beta}}+\mathbf{C} \Sigma_{\eta_{\beta} \eta_{\beta}} \mathbf{C}^{t}+\mathbf{C} \Sigma_{r_{\beta}^{\varepsilon} r_{\beta}^{\epsilon}} \mathbf{C}+\Sigma_{\varepsilon_{\| \beta} r_{\beta}^{\varepsilon}} \mathbf{C}^{t}+\mathbf{C} \Sigma_{r_{\beta}^{\varepsilon} \varepsilon_{\| \beta}}
$$

The matrix $\Sigma_{\widehat{\varepsilon}_{\| \beta} \widehat{\varepsilon}_{\| \beta}}$ can be readily estimated from the times series of $\boldsymbol{s}_{\boldsymbol{\beta}}(\boldsymbol{\rho}, t)$. The noise covariance matrix $\Sigma_{\boldsymbol{\eta}_{\boldsymbol{\beta}} \boldsymbol{\eta}_{\boldsymbol{\beta}}}$ is estimated from the temporal auto-correlation function of $\boldsymbol{s}_{\boldsymbol{\beta}}$. To determine the covariance matrix of the aliasing $\Sigma_{\boldsymbol{r}_{\boldsymbol{\beta}}^{\varepsilon} \boldsymbol{r}_{\boldsymbol{\beta}}^{\varepsilon}}$ and the cross-term $\Sigma_{\boldsymbol{\varepsilon}_{\| \boldsymbol{\beta}} \boldsymbol{r}_{\boldsymbol{\beta}}^{\varepsilon}}$, we consider Eq. 16. The covariance matrix $\Sigma_{\boldsymbol{r}_{\boldsymbol{\beta}}^{\varepsilon} \boldsymbol{r}_{\boldsymbol{\beta}}^{\varepsilon}}=\left\langle\boldsymbol{r}_{\boldsymbol{\beta}}^{\varepsilon} \boldsymbol{r}_{\boldsymbol{\beta}}^{\varepsilon}{ }^{t}\right\rangle$ is derived from the following expression :

$$
\Sigma_{\boldsymbol{r}_{\beta}^{\varepsilon} \boldsymbol{r}_{\beta}^{\varepsilon}}=\Sigma_{\boldsymbol{r}_{\beta} \boldsymbol{r}_{\beta}}+\mathbf{R} \Sigma_{\boldsymbol{r}_{\alpha} \boldsymbol{r}_{\alpha}} \mathbf{R}^{t}-\Sigma_{\boldsymbol{r}_{\beta} \boldsymbol{r}_{\alpha}} \mathbf{R}^{t}-\mathbf{R} \Sigma_{\boldsymbol{r}_{\alpha} \boldsymbol{r}_{\boldsymbol{\beta}}}
$$

where the matrix $\Sigma_{\boldsymbol{r}_{\beta} \boldsymbol{r}_{\alpha}}$ is the concatenation of the covariance matrices of off/on axis aliasing in loop disengaged. To determine the cross-term $\Sigma_{\boldsymbol{\varepsilon}_{\| \boldsymbol{\beta}} \boldsymbol{r}_{\boldsymbol{\beta}}^{\varepsilon}}$, we consider Eq. 10 that gives the analytic expression of $\boldsymbol{\varepsilon}_{\boldsymbol{\beta}}$. Assuming the independence of terms given in Eq. 10, the TS aliasing $\boldsymbol{r}_{\boldsymbol{\beta}}^{\boldsymbol{\varepsilon}}$ is only correlated with $\varepsilon_{\| \boldsymbol{\beta}}$. We thus have :

$$
\Sigma_{\varepsilon_{\| \beta} r_{\beta}^{\varepsilon}} \simeq \mathbf{C}\left\langle\mathbf{R} r_{\alpha} r_{\beta}^{\varepsilon t}\right\rangle \mathbf{C}^{t}
$$

Combining Eq. 28 with Eq. 16 leads thus to:

$$
\begin{aligned}
\Sigma_{\varepsilon_{\| \beta} \boldsymbol{r}_{\beta}^{\varepsilon}} & \simeq \mathbf{C}\left\langle\mathbf{R} \boldsymbol{r}_{\boldsymbol{\alpha}}\left(\boldsymbol{r}_{\boldsymbol{\beta}}-\mathbf{R} \boldsymbol{r}_{\boldsymbol{\alpha}}\right)^{t}\right\rangle \mathbf{C}^{t} \\
& \simeq \mathbf{C}\left(\mathbf{R} \Sigma_{\boldsymbol{r}_{\alpha} \boldsymbol{r}_{\boldsymbol{\beta}}}^{t}-\mathbf{R} \Sigma_{\boldsymbol{r}_{\alpha} \boldsymbol{r}_{\alpha}} \mathbf{R}^{t}\right) \mathbf{C}^{t}
\end{aligned}
$$

Finally, in combining Eq. 26, Eq. 27 and Eq. 29, one gets the final expression of $\Sigma_{\varepsilon_{\| \beta} \varepsilon_{\| \beta}}$

$$
\Sigma_{\varepsilon_{\| \beta} \varepsilon_{\| \beta}} \simeq \Sigma_{\widehat{\varepsilon}_{\| \beta} \widehat{\varepsilon}_{\| \beta}}-\mathbf{C} \Sigma_{\eta_{\beta} \eta_{\beta}} \mathbf{C}^{t}-\mathbf{C} \Sigma_{r_{\beta} r_{\beta}} \mathbf{C}^{t}+\mathbf{C R} \Sigma_{r_{\alpha} r_{\alpha}} \mathbf{R}^{t} \mathbf{C}^{t}
$$

Covariance matrices of aliasing required in Eq. 30 are derived analytically using the turbulence and system parameters, see paper 9909-163 in that conference.

\subsection{Determination of fitting and static OTFs}

\subsubsection{Static OTF, OTF Static $_{\beta}$}

In addition to NCPA, we have to deal with common static aberrations $\left\langle\boldsymbol{a}_{\boldsymbol{\beta}}\right\rangle$ in the science directions. These are not strongly mitigated by the loop as it is done for closed-loop systems. In MOAO, they must be calibrated on-sky and compensated by a DM offset. Even if the science object are too faint for WF sensing, the CANARY experiment has demonstrated it is feasible to offset the telescope towards a bright enough star to get the required calibration $\left({ }^{16}\right)$.

For MOAO PSF-R purpose, we determine $\left\langle\boldsymbol{a}_{\boldsymbol{\beta}}\right\rangle$ from the TS measurements using the Zernike reconstruction matrix $\mathbf{C}$, and considering noise and aliasing as zero-mean processes, as follows:

$$
\left\langle\boldsymbol{a}_{\boldsymbol{\beta}}\right\rangle=\mathbf{C}\left\langle\boldsymbol{s}_{\boldsymbol{\beta}}\right\rangle .
$$


Equation 17 involves a static $\mathrm{OTF}_{\mathrm{Static}_{\beta}}$ term. To avoid including multiple times the telescope OTF which is factored in every term as well as in Eq. 31, precaution must be taken to divide the OTFs by that of the telescope ${ }^{17}$ by doing :

$$
\operatorname{OTF}_{\operatorname{Static}_{\beta}}(\boldsymbol{\rho} / \lambda)=\frac{\iint_{\mathcal{P}} P(\boldsymbol{r}) P(\boldsymbol{r}+\boldsymbol{\rho}) \times e^{-i\left\langle\boldsymbol{a}_{\boldsymbol{\beta}}\right\rangle^{2}(\boldsymbol{r})} \times e^{-i\left\langle\boldsymbol{a}_{\boldsymbol{\beta}}\right\rangle^{2}(\boldsymbol{\rho}+\boldsymbol{r})} d \boldsymbol{r}}{\mathrm{OTF}_{\mathrm{tel}}(\boldsymbol{\rho} / \lambda)},
$$

where :

$$
\mathrm{OTF}_{\text {tel }}(\boldsymbol{\rho} / \lambda)=\iint_{\mathcal{P}} P(\boldsymbol{r}) P(\boldsymbol{r}+\boldsymbol{\rho}) d \boldsymbol{r}
$$

and applying a threshold for values of $\boldsymbol{\rho}$ nearing the telescope pupil.

\subsubsection{Determination of $\mathrm{OTF}_{\perp \beta}$}

The DM fitting $\mathrm{OTF}_{\perp \beta}$ is computed analytically from the perpendicular phase structure function that comes from the Power Spectrum Density (PSD) of spatial frequencies above the DM cut-off frequency, which scales with $\left(d / r_{0}\right)^{5 / 3}$. The proportionality has been calibrated using end-to-end simulations $\left({ }^{11}\right)$. We get $\sigma_{\text {Fitting }}^{2}=$ $0.3125 \times\left(d / r_{0}\right)^{5 / 3}$ for the CANARY Adonis DM.

\section{PSF-R RESULTS USING ON-SKY CANARY DATA}

\subsection{Statistics on reconstruction performance}

Figure 3 depicts results on reconstructed Strehl-ratio (SR) and Full Width at Half Maximum (FWHM) as function of the corresponding sky quantities. Each point is a result of the PSF-R performed on a single data set acquired in September 13, 15, 17 and 18 2013, where CANARY was operating is phase B configuration, observing a four stars asterism, with a central bright star and with four Rayleigh LGS focused at $21 \mathrm{~km}$ (see. ${ }^{12}$ for observation details). We report in Tab. 2 statistics of the reconstruction in terms of SR and FWHM.

The FWHM is determined from fitting the reconstructed PSF to five-parameters Moffat function :

$$
f\left(x, y, I_{0}, \alpha_{x}, \alpha_{y}, \beta, \theta\right)=I_{0}\left(1+\left(\frac{x \cos (\theta)-y \sin (\theta)}{\alpha_{x}}\right)^{2}+\left(\frac{x \sin (\theta)-y \cos (\theta)}{\alpha_{y}}\right)^{2}\right)^{-\beta},
$$

where $I_{0}$ is the PSF amplitude, $\alpha_{x}$ and $\alpha_{y}$ coefficients of spreading along $x$ and $y$ directions, $\beta$ the skewness coefficient and $\theta$ the rotation coefficient. The FWHM is then given by :

$$
\mathrm{FWHM}=\left(2 \times\left(2^{1 / \beta}-1\right) \times\left(\alpha_{x}^{2}+\alpha_{y}^{2}\right)\right)^{1 / 2} .
$$

We obtained $95 \%$ of correlation on the SR over 450 data sets with TS-based method - table 2. The reconstruction is affected by a bias of $0.41 \%$ (SR are ranged between 0 and $100 \%$ here), which is under the error bars of few percent on the H-band SR estimation directly on the science images. In addition, we get a 1- $\sigma$ standard deviation of $3.52 \%$ of SR, over a large swath of observation conditions. In terms of FWHM, we get $92.5 \%$ of correlation, a bias of 2.71 mas and a 1- $\sigma$ standard deviation of 14.9 mas. On the WHT, we get about 80 mas for $\lambda / D$ in H-band. The high correlation obtained on SR and FWHM with the reconstructed PSFs confirm the accurateness of the profiling estimation routines and system calibration.

From table. 2, the RTC-based method achieves satisfactory results considering the approximations done to determine the PSF when comparing with the TS-based method. On the SR, we get $92.3 \%$ of correlation, a bias of $1.29 \%$ and a $1-\sigma$ standard-deviation of $4.82 \%$. On the FWHM, we get $88.3 \%$ of correlation, a bias of 2.94 mas and a $1-\sigma$ standard deviation of 19.9 mas. As noticed on Fig. 3, we get a larger scattering than using the TS-based method. The higher bias with the RTC-based method than with the TS-based cannot be properly interpreted since those bias are smaller than the expected errors bars on the sky SR estimation.

An important point to notice is the FWHM is similarly well reconstructed with both methods. It means the static aberrations, that spread out the PSF core and contributes significantly to the FWHM, are well taken into account and well calibrated. It means methods developed for static aberrations calibration are robust and accurate enough to reach the level of PSF reconstruction ensured with the TS-based method. 

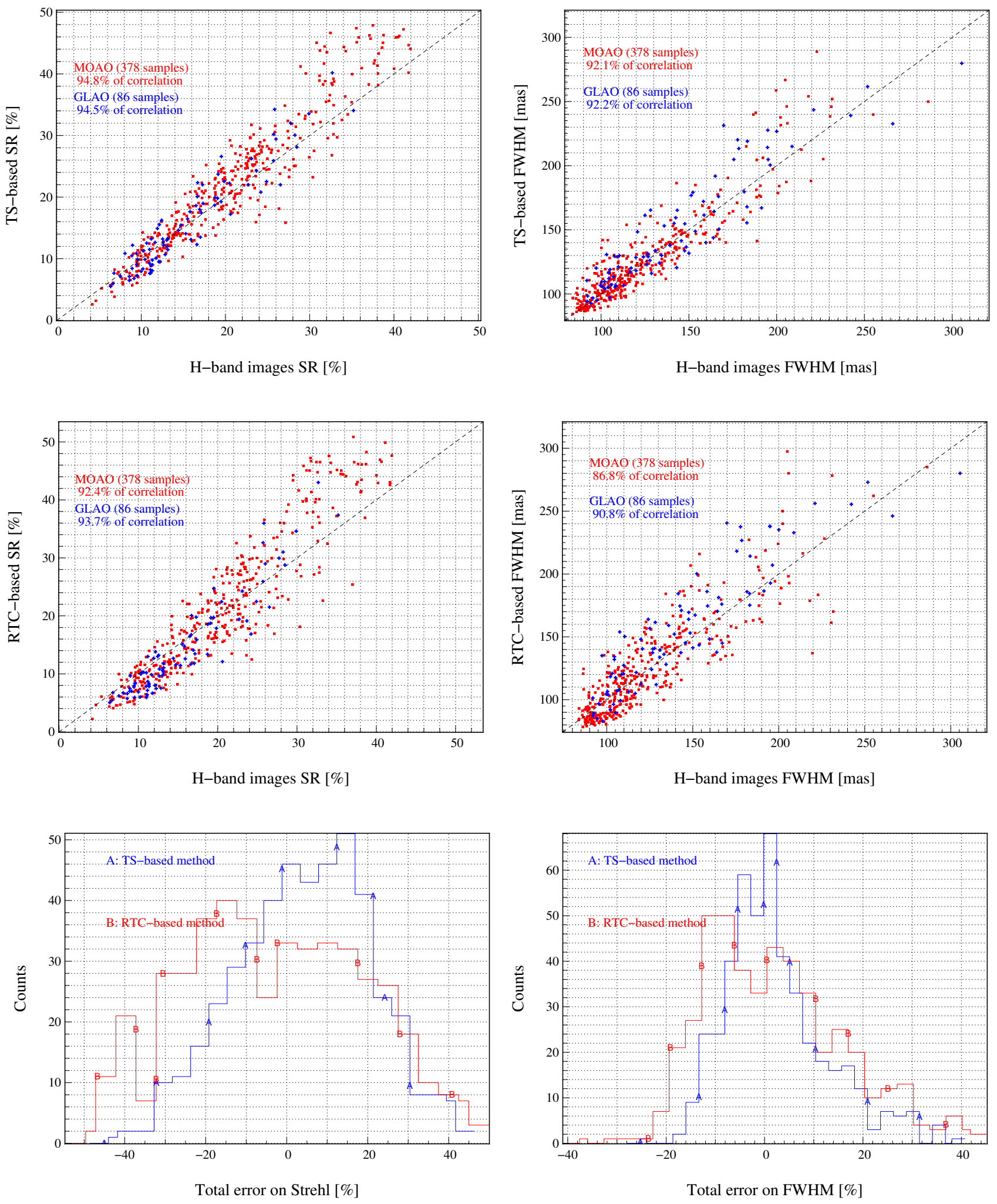

Figure 3: Left: TS-based/RTC-based reconstructed SR as function of H-band images SR. and histograms of the relative error on the SR. Right: TS-based/RTC-based reconstructed FWHM as function of H-band images FWHM. and histograms of the relative error on the FWHM. 


\begin{tabular}{|l||c|c|c|c|}
\hline \multicolumn{1}{|c|}{ Method } & Criteria & Mean & Std & Correlation [\%] \\
\hline \multirow{2}{*}{ RTC-based } & $\Delta \mathrm{SR}=\mathrm{SR}_{\text {rtc }}-\mathrm{SR}_{\text {sky }}[\%]$ & 1.29 & 4.82 & 92.3 \\
& $\Delta \mathrm{FWHM}=\mathrm{FWHM}_{\mathrm{rtc}}-\mathrm{FWHM}_{\text {sky }}[\mathrm{mas}]$ & 2.94 & 19.9 & 88.3 \\
\hline \multirow{2}{*}{ TS-based } & $\Delta \mathrm{SR}=\mathrm{SR}_{\mathrm{ts}}-\mathrm{SR}_{\text {sky }}[\%]$ & 0.41 & 3.52 & 95.0 \\
& $\Delta \mathrm{FWHM}=\mathrm{FWHM}_{\mathrm{ts}}-\mathrm{FWHM}_{\text {sky }}[\mathrm{mas}]$ & 2.71 & 14.9 & 92.5 \\
\hline
\end{tabular}

Table 2: Statistics on PSF-R performed using either the RTC-based or the TS-based method. They are given for an amount of 450 CANARY MOAO data sets acquired in September 2013. SR are ranged between 0 and $100 \%$.

\subsection{Data set 00h15m36s on September 132013}

We focus now on a particular data set acquired at 00h15m36s on September 13th 2013. CANARY was operating in MOAO mode based on four LGS and three NGS. During 2048 temporal frames, sampled at $150 \mathrm{~Hz}$, the RTC has provided synchronized telemetry coming from WFS and imaging camera.

We report in Fig. 4 both sky and reconstructed PSFs. On the sky PSF, we measure a H-band SR of $24.0 \%$ and a FWHM of 107.5 mas. With the Ts-based method, with a SR of $24.05 \%$ and a FWHM of 111.4 mas. Then, with the RTC-based method, we reach $24.5 \%$ of SR and 118.8 mas of FWHM. The TS-based method achieves $0.2 \%$ and $3.4 \%$ of relative error on respectively the SR and FWHM reconstruction, while the RTCbased method reaches $2 \%$ and $10.5 \%$ of relative error. In addition, Fig. 4 illustrates the PSF core elongation is well reconstructed whatever the method.
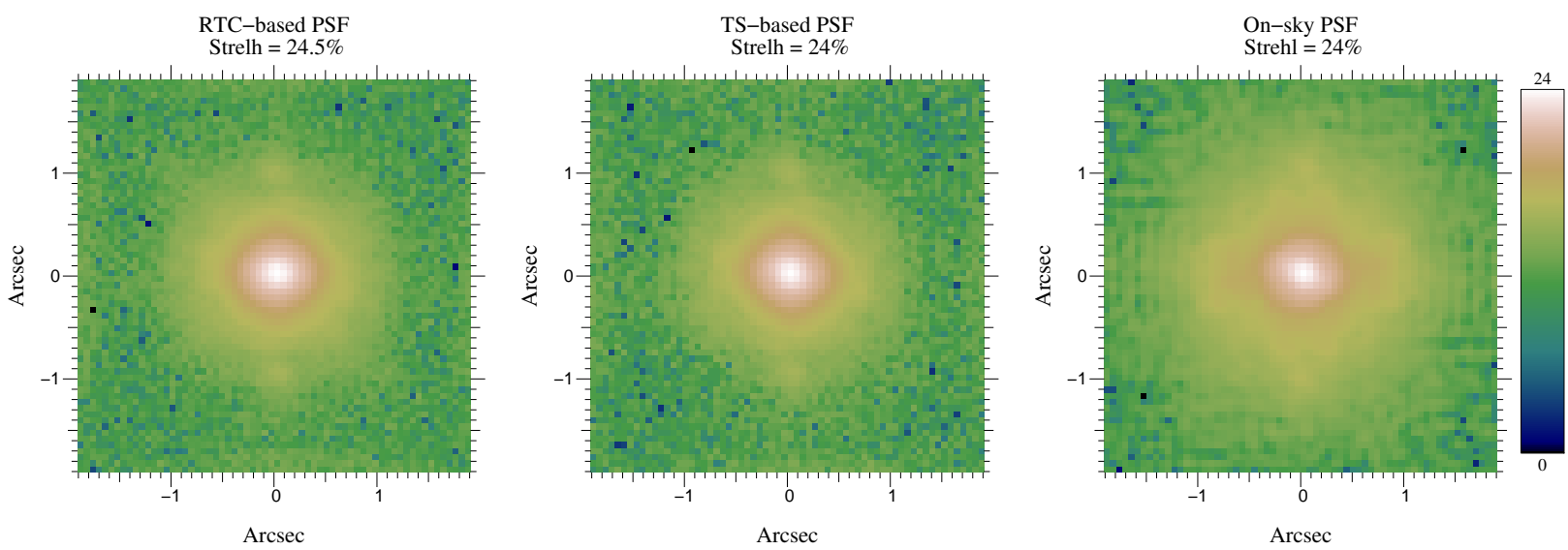

Figure 4: From left to right: reconstructed PSF using the RTC-based method, the TS-based and acquired on-sky in H-band (logarithmic scale). The reconstructed PSFs include an additional Gaussian noise whose the standard-deviation is measured from the corner of the sky PSF, outside the inner tangent circle to the edges of the image.

In Fig.5 we report the relative error on the Ensquared Energy (EE), the radial average of the PSFs and the OTFs. One can notice the quality of the reconstruction is not only a matter of SR and FWHM. We stay below $6 \%$ of relative error on the EE. On the OTF, we reconstruct more energy from the low spatial frequencies that translates a faster decreasing of the reconstructed PSF core than the sky, as we noticed on the radial PSF curves between 0.2 and 0.5 arcsec. We believe it is related to assumptions on the DM: the phase is represented by Zernike modes and not by DM influence functions. This effect is minimized when using a reconstruction matrix calibrated on the bench. 

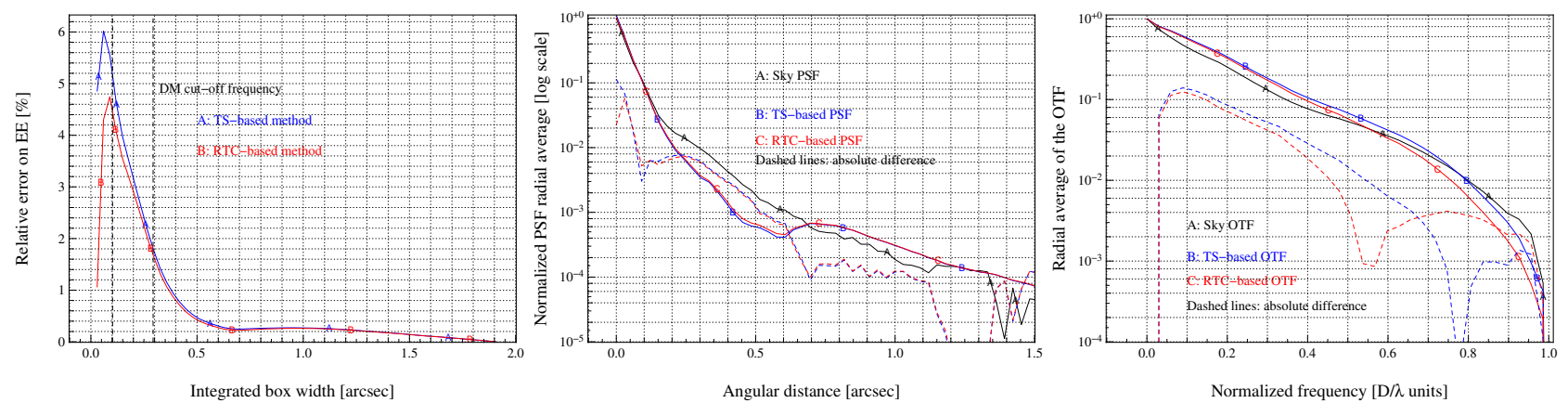

Figure 5: Top-Left:From left to right Relative error on the Ensquared Energy computed on the reconstructed PSF. Radial average of both reconstructed/sky PSFs as function of the angular separation from center. Radial average of both reconstructed/sky OTFs as function of the normalized frequency.

\section{CONCLUSIONS}

We have laid out a PSF-R algorithm tailored to open-loop, tomographic MOAO systems and compared it to a reference method using the Truth-Sensor and to on-sky metrics (SR, FWHM, EE) obtained directly from the science detector.

We have processed over 450 data sets of telemetry from the CANARY MOAO demonstrator. We report $94 \%$ and $95 \%$ of correlation between the reconstructed and sky SR in using respectively the RTC-based and TSbased method. On the FHWM, we have respectively $90 \%$ and $94 \%$ of correlation. Bias on reconstructed values are lower than errors bars on sky values for both methods on both SR and FWHM. We get also very similar standard-deviation on reconstructed values as well for both methods.

This work shows that fine-detailed system calibration and modeling can be operated to achieve such high levels of correlation on SR and FWHM. We emphasis particularly on the reliability and efficiency of tools implemented for turbulence characterization based on the Learn \& Apply approach (see paper 9909-163), and static aberrations calibration as well, since we are able to reproduce the asymmetric shape of the PSF caused by these static aberrations.

We now plan to improve the reconstruction method by reviewing the approximations/assumptions by evaluating cross-terms using end-to-end simulations and by integrating measurable matrices from the bench on the reconstruction process. For instance, we can tackle bias and standard-deviation on FWHM estimation in using directly the DM influence functions calibrated on bench.

We will then extend results to ELT-sized systems particularly in view of the LTAO instrument Harmoni. We aim to overview existing PSF reconstruction methods and compare each other to the RTC-based approach in terms of accuracy of reconstruction, and describe which one is more tailored for a given science case.

\section{ACKNOWLEDGMENTS}

The research leading to these results received the support of the A*MIDEX project (no. ANR-11-IDEX-0001-02) funded by the Investissements d'Avenir French Government program, managed by the French National Research Agency (ANR). This work is also supported CNRS, INSU, Observatoire de Paris, Université Paris Diderot-Paris 7 and European Commission (Fp7 Infrastructures 2012-1, OPTICON Grant 312430, WP1).

\section{REFERENCES}

[1] Veran, J.-P., Rigaut, F., Maitre, H., and Rouan, D., "Estimation of the adaptive optics long-exposure pointspread function using control loop data.," Journal of the Optical Society of America A 14, 3057-3069 (Nov. 1997). 
[2] Cuby, J.-G., Morris, S., Bryson, I., Lehnert, M., Evans, C., Fusco, T., Jagourel, P., Myers, R., Rousset, G., Schnetler, H., Amans, J.-P., Allington-Smith, J., Assemat, F., Beard, S., Chemla, F., Content, R., Dipper, N., Ferrari, M., Gendron, E., Gimenez, J.-L., Hastings, P., Hubert, Z., Hugot, E., Laporte, P., Leroux, B., Madec, F., Neichel, B., Morris, T., Prieto, E., Swinbank, M., Talbot, G., Taylor, W., Vidal, F., Vivès, S., Vola, P., and Wells, M., [EAGLE: an MOAO fed multi-IFU in the NIR on the E-ELT], vol. 7014 of Proc. SPIE (Aug. 2008).

[3] McPherson, A., Spyromilio, J., Kissler-Patig, M., Ramsay, S., Brunetto, E., Dierickx, P., and Cassali, M., [E-ELT update of project and effect of change to 39m design], vol. 8444 of Proc. SPIE (Sept. 2012).

[4] Evans, C. J., Lehnert, M. D., Cuby, J.-G., Morris, S. L., Swinbank, A. M., Taylor, W. D., Alexander, D. M., Lorente, N. P. F., Clénet, Y., and Paumard, T., [Science requirements for EAGLE for the E-ELT], vol. 7014 of Proc. SPIE (Aug. 2008).

[5] Hammer, F., Barbuy, B., Cuby, J. G., Kaper, L., Morris, S., Evans, C. J., Jagourel, P., Dalton, G., Rees, P., Puech, M., Rodrigues, M., Pearson, D., and Disseau, K., [MOSAIC at the E-ELT: A multi-object spectrograph for astrophysics, IGM and cosmology], vol. 9147 of Proc. SPIE , 27 (Aug. 2014).

[6] Vernet, E., Cayrel, M., Hubin, N., Mueller, M., Biasi, R., Gallieni, D., and Tintori, M., [Specifications and design of the E-ELT M4 adaptive unit], vol. 8447 of Proc. SPIE (July 2012).

[7] Andersen, D. R., Fischer, M., Conan, R., Fletcher, M., and Véran, J., [VOLT: the Victoria Open Loop Testbed], vol. 7015 of Proc. SPIE (July 2008).

[8] Gavel, D., Severson, S., Bauman, B., Dillon, D., Reinig, M., Lockwood, C., Palmer, D., Morzinski, K., Ammons, M., Gates, E., and Grigsby, B., [Villages: an on-sky visible wavelength astronomy AO experiment using a MEMS deformable mirror], vol. 6888 of Proc. SPIE (Mar. 2008).

[9] Gendron, E., Vidal, F., Brangier, M., Morris, T., Hubert, Z., Basden, A., Rousset, G., Myers, R., Chemla, F., Longmore, A., Butterley, T., Dipper, N., Dunlop, C., Geng, D., Gratadour, D., Henry, D., Laporte, P., Looker, N., Perret, D., Sevin, A., Talbot, G., and Younger, E., "MOAO first on-sky demonstration with CANARY," Astron. 83 Astrophys. 529, L2 (May 2011).

[10] Lavigne, J.-F., Lamontagne, F., Anctil, G., Wang, M., Tremblay, M., Lardière, O., Nash, R., Andersen, D., Savard, M., Côté, P., Bradley, C. H., and Châteauneuf, F., "Design and test results of the calibration unit for the MOAO demonstrator RAVEN," in [Adaptive Optics Systems III], Proc. SPIE 8447, 844754 (July 2012).

[11] Vidal, F., Gendron, É., Rousset, G., Morris, T., Basden, A., Myers, R., Brangier, M., Chemla, F., Dipper, N., Gratadour, D., Henry, D., Hubert, Z., Longmore, A., Martin, O., Talbot, G., and Younger, E., "Analysis of on-sky MOAO performance of CANARY using natural guide stars," Astron. 8 Astrophys. 569, A16 (Sept. 2014).

[12] Morris, T., Gendron, E., Basden, A., Martin, O., Osborn, J., Henry, D., Hubert, Z., Sivo, G., Gratadour, D., Chemla, F., Sevin, A., Cohen, M., Younger, E., Vidal, F., Wilson, R., Butterley, T., Bitenc, U., Reeves, A., Bharmal, N., Raynaud, H.-F., Kulcsar, C., Conan, J.-M., Huet, J.-M., Perret, D., Dickson, C., Atkinson, D., Bailie, T., Longmore, A., Todd, S., Talbot, G., Morris, S., Rousset, G., and Myers, R., [CANARY phase B: on-sky open-loop tomographic LGS AO results], vol. 9148 of Proc. SPIE, 1 (July 2014).

[13] Vidal, F., Gendron, E., and Rousset, G., "Tomography approach for multi-object adaptive optics," JOSA A 27, A253-A264 (Nov. 2010).

[14] Martin, O., Démonstration de l'optique adapative multi-objet avec étoiles lasers par Canary, PhD thesis, Université Paris DIderot (2014).

[15] Gendron, E., Morel, C., Osborn, J., Martin, O., Gratadour, D., Vidal, F., Le Louarn, M., and Rousset, G., [Robustness of tomographic reconstructors versus real atmospheric profiles in the ELT perspective], vol. 9148 of Proc. SPIE , 4 (Aug. 2014).

[16] Gratadour, D andGendron, E., Grosset, L., Morris, T., Osborn, J., Basden, A., Martin, O., Rouan, D andMyers, R., and Rousset, G., "First demo science with MOAO: observations of distant merging galaxies with CANARY," in [Proceedings of the Third AO4ELT Conference.], (2013).

[17] Gendron, E., Clénet, Y., Fusco, T., and Rousset, G., "New algorithms for adaptive optics point-spread function reconstruction," Astron. \&3 Astrophys. 457, 359-363 (Oct. 2006). 